\section{Campagne contre l'acci- dent vasculaire cérébral de la Fondation suisse de cardiologie (SHS) et du Groupe suisse de travail pour les maladies cérébro-vasculaires (ZAS)}

Editorial et conseils pratiques

\section{J. Bogousslavsky ${ }^{a}$, W. Rutishauser ${ }^{b}$, H. Mattle}

a CHUV, Lausanne

Membre du Groupe de travail suisse des maladies cérébro-vasculaires et président du Conseil Européen des Attaques cérébrales ("European Stroke Council»)

${ }^{\mathrm{b}}$ HUG, Genève

Président de la Fondation suisse de cardiologie

Hôpital de l'Ile, Berne

Membre du Groupe suisse de travail sur les maladies cérébro-vasculaires et dirigeant de la campagne d'information

La campagne 2000 de la Fondation suisse de cardiologie est dédiée à l'infarctus cérébral, un problème médical et social important. Durant l'année 2000, plus de 12000 personnes habitant en Suisse souffriront d'un infarctus cérébral. Malgré la mortalité très basse de l'infarctus cérébral en Suisse, comparée aux données internationales, 20 à 30\% des patients suisses meurent et 40 à 50\% gardent à long terme une invalidité importante. $7 \%$ des retraités suisses (>65 ans) ont souffert d'un infarctus cérébral. Pour cette raison, la prévention primaire, la reconnaissance précoce des symptômes d'alerte, le traitement aigu optimal, ainsi que la prévention secondaire de cette maladie ont une importance primordiale. La Fondation suisse de cardiologie et le groupe suisse de travail pour les maladies cérébro-vasculaires se sont réunis pour mener une campagne d'information sur les infarctus cérébraux. La Fondation suisse de cardiologie a été fondée en 1976 par quelques cardiologues en présence de l'augmentation des maladies cardio-vasculaires. Son but est de faire avancer la recherche scientifique de même que l'information de la population. Le Groupe suisse de travail sur les maladies cérébro-vasculaires est une association de médecins et de scientifiques fondée en 1995, s'intéressant à la clinique et la recherche sur les maladies cérébro-vasculaires. La majorité de ses membres sont des neurologues, des neurochirurgiens et des neuroradiologues.

\section{Figure 1}

"L'accident vasculaire cérébral - connaître les risques, saisir les chances». Logo de la campagne contre l'accident vasculaire cérébral de la Fondation Suisse de Cardiologie et du Groupe suisse de travail pour les maladies cérébro-vasculaires.

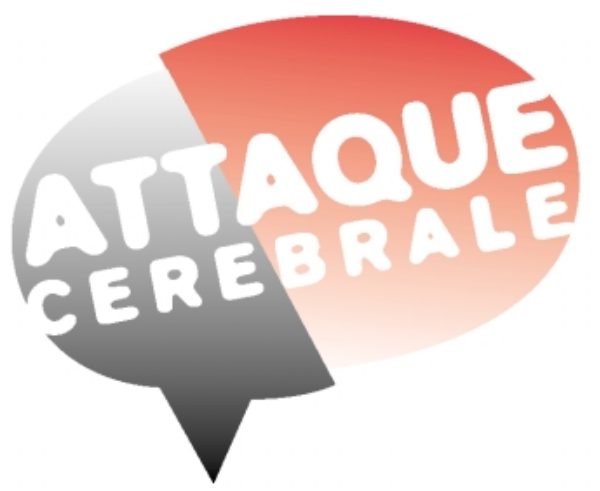

connaître les risques saisir les chances

Cette campagne de prévention «accident vasculaire cérébral - connaître les risques, saisir les chances» est une première, et veut se concentrer, selon ses deux groupes initiateurs sur la reconnaissance des symptômes et un comportement correct lors d'une urgence (fig 1). Diverses activités à l'attention du public sont prévues: un test de risque facilement réalisable, qui sera évalué par informatique et retourné à la personne au moyen d'un rapport individualisé dans une lettre de deux pages. De plus, des conférences de vulgarisation sont prévues dans toute la Suisse, avec des actions de dépistage ("screening") dans les grandes surfaces au moyen de stands d'information et d'évaluation techniquement avancée. Toutes ces actions seront accompagnées d'une information intensive à travers les médias. Une ligne téléphonique de conseil sera établie par la Fondation suisse de cardiologie et la Société suisse de cardiologie, gérée par des médecins, et à disposition de la population pendant la durée entière de cette campagne de 2 ans. Une brochure sur l'infarctus cérébral sera crée pour l'AI.

L'orientation des médecins se fera par un mailing pour la campagne et une série d'articles spécifiques dans le Bulletin des médecins suisses. Ces articles spéciaux peuvent être obtenus sous forme reliée à la Fondation suisse de cardiologie. Un dossier de diapositives et de transparents sera à disposition sur demande pour des conférences. Le succès de cette campagne dépendra surtout de l'acceptation et du soutien par les médecins suisses, à partir de ces informations de base.

Le lancement de la campagne est prévu pour mai 2000. Une information préalable chez des parlementaires suisses, avec le test de dépistage mentionné cidessus, est prévue. Les résultats seront à disposition pour la campagne. 


\section{Qu'est-ce un accident vasculaire cérébral?}

Un accident vasculaire cérébral est généralement l'expression d'une perfusion cérébrale focale insuffisante (infarctus cérébral) suite à une occlusion athérothrombotique ou embolique d'une artère cérébrale, plus rarement déclenché par une hémorragie. Une bonne nouvelle est que nous pouvons tous nous protéger contre la survenue d'un accident vasculaire cérébral en connaissance de cause, en modifiant et traitant ses facteurs de risque, en faisant attention à ses signes d'alerte, et en sachant quoi faire lors de sa survenue.

L'athéromatose et la thrombose sont la base des maladies circulatoires en général, touchant le cerveau (infarctus cérébral) le cœur (angine de poitrine, infarctus du myocarde) ou des vaisseaux périphériques (claudication). Les facteurs de risque vasculaires, qui sont à l'origine de ces maladies, sont globalement identiques pour tous les territoires vasculaires:

- hypertension artérielle;

- tabagisme;

- diabète;

- hyperlipidémie;

- surconsommation d'alcool;

- certains troubles cardiaques, notamment la fibrillation auriculaire;

- syndrome d'apnée du sommeil.

\section{Que pouvons-nous faire dans la vie courante?}

- Arrêter le tabagisme pour les fumeurs;

- avoir une activité physique, régulière et abondante;

- avoir des habitudes de nutrition saines (régime méditerranéen) et contrôle du poids;

- éviter la surconsommation d'alcool et la consommation de drogues.

\section{Comment le médecin peut-il traiter préventivement?}

- Traitement médicamenteux de l'hypertension artérielle;

- traitement multifactoriel du diabète;

- traitement de l'hyperlipidémie par des mesures nutritionnelles, éventuellement des statines;

- anticoagulation correcte lors de fibrillation auriculaire et valve cardiaque artificielle;

- investigations d'un souffle carotidien;

- inhibiteurs de l'agrégation thrombocytaire pour la prévention secondaire dans la majorité des patients;

- traitement du syndrome d'apnée du sommeil par des mesures spécifiques (CPAP).

\section{"Minuit moins cinq"}

2/3 des infarctus cérébraux représentent un événement nouveau alors qu'un $1 / 3$ survient chez des patients qui ont déjà présenté un infarctus cérébral ou un accident ischémique transitoire (AIT). Une prévention primaire ou secondaire optimale individualisée peut diminuer le nombre d'infarctus cérébraux de 30 à $50 \%$. La reconnaissance précoce de signes annonciateurs et d'alerte est indispensable. 2500 à 3000 suisses souffrent d'AIT chaque année. Ces AIT sont des "mini-infarctus cérébraux", pendant lesquels les thrombi plaquettaires provoquent des déficits visuels, du langage, de la compréhension, de la sensibilité, ou de la force de l'hémivisage, d'un bras ou d'une jambe, ou de l'hémicorps durant peu de temps. Une sténose carotidienne ou une athéromatose extensive de l'aorte sont souvent à l'origine de ces AIT. Ces patients ont besoin d'une recherche étiologique et d'une prévention secondaire ciblée les jours suivant le premier AIT.

\section{L'infarctus cérébral aigu}

Une symptomatologie dépassant la durée typique d'un AIT de 8 à 14 minutes représente en général un événement ischémique important avec infarctus cérébral. Dans ces cas, le contact immédiat avec un médecin ou un appel au numéro d'urgence 144 devient décisif pour l'individu concerné, afin qu'il soit dirigé vers un hôpital bien équipé, possédant si possible un "stroke team». Chaque infarctus cérébral doit être considéré comme une urgence prioritaire. Un traitement intensif 3 heures après le début des symptômes peut diminuer les séquelles d'un infarctus cérébral d'une manière significative. Une évaluation radiologique rapide permet de distinguer une ischémie cérébrale d'une hémorragie. Dans le cas d'une occlusion thrombotique, une thrombolyse immédiate représente un traitement efficace, toutefois liée à quelques risques. Le taux d'invalidité significatif peut être abaissé de $40-50 \%$ à 25-35\% avec la thrombolyse.

Après la phase aiguë de l'infarctus cérébral commence la phase de la convalescence et de la rééducation. Une équipe formée de médecins, d'infirmières, de physiothérapeutes et d'autres spécialistes collabore étroitement entre eux et avec la famille du patient. La volonté et la motivation permanente du patient lui permettent de compenser et de regagner les fonctions perdues et sont décisives. Le but de cette rééducation est toujours de permettre au patient de mener une vie indépendante. La famille est un facteur extrêmement important dans le soutien du patient. Même les améliorations les plus discrètes sont des étapes importantes pour le patient. L'encouragement, le soutien et l'assurance des proches peuvent être utiles au bienêtre du patient. 
Les connaissances actuelles sur les infarctus cérébraux seront publiées dans le bulletin des médecins suisses dans des articles hebdomadaires. La première partie sous la responsabilité du Professeur H. Mattle (Berne) traite de l'infarctus cérébral aigu, le diagnostic et le traitement. La deuxième partie sous la responsabilité du PD Dr P. Lyrer (Bâle) traite de l'épidémiologie et des facteurs de risque. Dans une troisième série d'articles coordonnés par le PD R. Baumgartner (Zurich), la rééducation et la prévention secondaire sont discutées. La quatrième et dernière série, sous la responsabilité du Dr A. Barth (Berne) concerne les hémorragies intracrâniennes non-traumatiques.

Le but de cette série d'articles est d'informer les médecins suisses des progrès dans les connaissances sur l'accident vasculaire cérébral. Le groupe suisse de travail pour les maladies cérébro-vasculaires et la Fondation suisse de cardiologie espèrent pouvoir diminuer les conséquences majeures des maladies cérébro-vasculaires par cette campagne d'information 2000. 